\title{
Large guanidinium cation mixed with methylammonium in lead iodide perovskites for $19 \%$ efficient solar cells
}

\author{
Alexander D. Jodlowski ${ }^{1,2}$, Cristina Roldán-Carmona ${ }^{1 \star}$, Giulia Grancini', Manuel Salado ${ }^{1,3}$, \\ Maryline Ralaiarisoa ${ }^{4,5}$, Shahzada Ahmad ${ }^{3}$, Norbert Koch ${ }^{14,5}$, Luis Camacho ${ }^{2}$, Gustavo de Miguel ${ }^{2 \star}$ \\ and Mohammad Khaja Nazeeruddin ${ }^{1{ }^{1 \star}}{ }^{1 \star}$
}

Organic-inorganic lead halide perovskites have shown photovoltaic performances above $20 \%$ in a range of solar cell architectures while offering simple and low-cost processability. Despite the multiple ionic compositions that have been reported so far, the presence of organic constituents is an essential element in all of the high-efficiency formulations, with the methylammonium and formamidinium cations being the sole efficient options available to date. In this study, we demonstrate improved

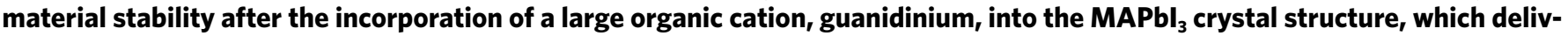
ers average power conversion efficiencies over 19\%, and stabilized performance for 1,000 $\mathrm{h}$ under continuous light illumination, a fundamental step within the perovskite field.

(1) ybrid perovskites have recently emerged as outstanding materials for efficient and low-cost solar technology. Unique properties, including high absorption coefficient ${ }^{1}$, large charge-carrier diffusion length ${ }^{2}$, small exciton binding energy ${ }^{3}$ and low trap density ${ }^{4}$, pushed their performances to a record efficiency beyond $22 \%{ }^{5}$. The most common perovskite arranges into $\mathrm{APbX}_{3}$, $\left(\mathrm{A}=\mathrm{CH}_{3} \mathrm{NH}_{3}{ }^{+}\right.$(methylammonium, $\mathrm{MA}$ ) or $\mathrm{CH}_{3}\left(\mathrm{NH}_{2}\right)_{2}{ }^{+}$(formamidinium, $\mathrm{FA}) ; \mathrm{X}=\mathrm{Cl}^{-}, \mathrm{Br}^{-}$or $\left.\mathrm{I}^{-}\right)$, which crystallizes in a three-dimensional (3D) network ${ }^{3}$. Among different candidates, mixed ionic compositions of MA and FA cations ${ }^{6}$ and $\mathrm{Br}^{-} / \mathrm{I}^{-}$halides have recently led to superior performance, with efficiencies close to $21 \%$ for (FAP $\left.\mathrm{bI}_{3}\right)_{0.85}\left(\mathrm{MAPbBr}_{3}\right)_{0.15}$ (ref. $\left.{ }^{7}\right)$. Nevertheless, these improvements in the efficiency of perovskite materials come with stability concerns, due to decomposition to the initial precursors ${ }^{8}$, and intrinsic halide segregation ${ }^{9,10}$ with ambiguous long-term consequences.

To overcome the stability issue, a few strategies have been proposed $^{11-14}$, including the exploration of alternative cations. Recent reports have shown promising results with the introduction of $\mathrm{Cs}^{+}$ and $\mathrm{Rb}^{+}$mixtures into the hybrid $\left(\mathrm{FAPbI}_{3}\right)_{0.85}\left(\mathrm{MAPbBr}_{3}\right)_{0.15}$ structure, leading to a complex triple ${ }^{15}$ and quadruple ${ }^{16}$ composition with efficiency over $20 \%$. Nevertheless, organic alternatives with a proper ionic radius $(R)$, such as hydrazinium $\left[\mathrm{H}_{3} \mathrm{~N}-\mathrm{NH}_{2}\right]^{+}$or azetidinium $\left[\left(\mathrm{CH}_{2}\right)_{3} \mathrm{NH}_{2}\right]^{+}$(ref. $\left.{ }^{17}\right)$, that can fit into the inorganic $\mathrm{Pb}-\mathrm{I}$ framework while maintaining an adequate Goldschmidt tolerance factor $\left(t=\left(R_{\mathrm{A}}+R_{\mathrm{I}}\right) / \sqrt{2}\left(R_{\mathrm{Pb}}+R_{\mathrm{I}}\right)\right.$ to ensure a cubic structure $(t=0.8-1)^{17,18}$ are not available. On the other hand, obtaining good perovskite films with alternative cations has been experimentally challenging, and no results comparable to the state-of-the-art perovskites have been reported so far
Here we introduce a perovskite based on the organic cation guanidinium $\left(\mathrm{CH}_{6} \mathrm{~N}_{3}{ }^{+}\right.$, Gua $)$as a more stable and efficient alternative to the state-of-the-art $\mathrm{MAPbI}_{3}$. Gua features an ionic radius of $\sim 278 \mathrm{pm}$ (ref. ${ }^{17}$ ), slightly above the upper limit of the tolerance factor ( $t$ 1.03), forming low-dimensional perovskites (LDPs) when mixed with $\mathrm{PbI}_{2}$ (refs ${ }^{19,20}$ ). In this work, we demonstrate that when combined with methylammonium in a mixed $\mathrm{MA}_{1-x} \mathrm{Gua}_{x} \mathrm{PbI}_{3}$ composition $(0<x<0.25)$ the Gua cation inserts in the crystal unit, forming a $3 \mathrm{D}$ perovskite with enhanced thermal and environmental stability. Our results widen the exploration of cations with a radius beyond the tolerance limit, while preserving a $3 \mathrm{D}$ structure and high photovoltaic performance. With this approach, solar cells display an average photoconversion efficiency (PCE) of $19.2 \pm 0.4 \%$ at enhanced stability.

\section{Structural characterization of $\mathrm{MA}_{1-x} \mathrm{Gua}_{x} \mathrm{Pbl}_{3}$ perovskites} To gain insight into the arrangement of the Gua cations in the $\mathrm{MA}_{1-x} \mathrm{Gua}_{x} \mathrm{PbI}_{3}$ crystal structure, $\mathrm{X}$-ray diffraction (XRD) measurements of the thin films were performed (see Methods for details). Figure 1a shows the diffractograms of the perovskite films containing MA/Gua mixtures, where $0<x_{\text {Gua }}<1\left(x_{\text {Gua }}\right.$ represents the Gua molar ratio), compared with $\mathrm{MAPbI}_{3}$. The incorporation of Gua cations yields a gradual decrease of the reflection peak intensity, but retaining the $\mathrm{MAPbI}_{3}$ tetragonal phase even for $x_{\text {Gua }}=0.25$. Larger Gua percentages significantly decrease the diffraction signal, while the appearance of new diffraction peaks at $8.54^{\circ}$ and $11.31^{\circ}$ indicates the formation of a $1 \mathrm{D} \mathrm{GuaPbI}{ }_{3}$ phase, as previously reported ${ }^{21}$. Interestingly, a closer inspection of the reflection peaks reveals a notable shift to lower angles, as shown in the zoom of Fig. 1b

\footnotetext{
Group for Molecular Engineering of Functional Materials, Institute of Chemical Sciences and Engineering, Ecole Polytechnique Fédérale de Lausanne (EPFL), Sion, Switzerland. '2Departamento de Química Física y Termodinámica Aplicada, Instituto Universitario de Investigación en Química Fina y Nanoquímica IUQFN, Universidad de Córdoba, Campus de Rabanales, Edificio Marie Curie, Córdoba, Spain. ${ }^{3}$ Abengoa Research, Abengoa, C/ Energía Solar no. 1, Campus Palmas Altas, Sevilla, Spain. ${ }^{4}$ Humboldt-Universität zu Berlin, Institut für Physik \& IRIS Adlershof, Berlin, Germany. ${ }^{5}$ HelmholtzZentrum Berlin für Materialien und Energie, Devision Renewable Energies, Berlin, Germany. *e-mail: cristina.roldancarmona@epfl.ch; gmiguel@uco.es; mdkhaja.nazeeruddin@epfl.ch
} 


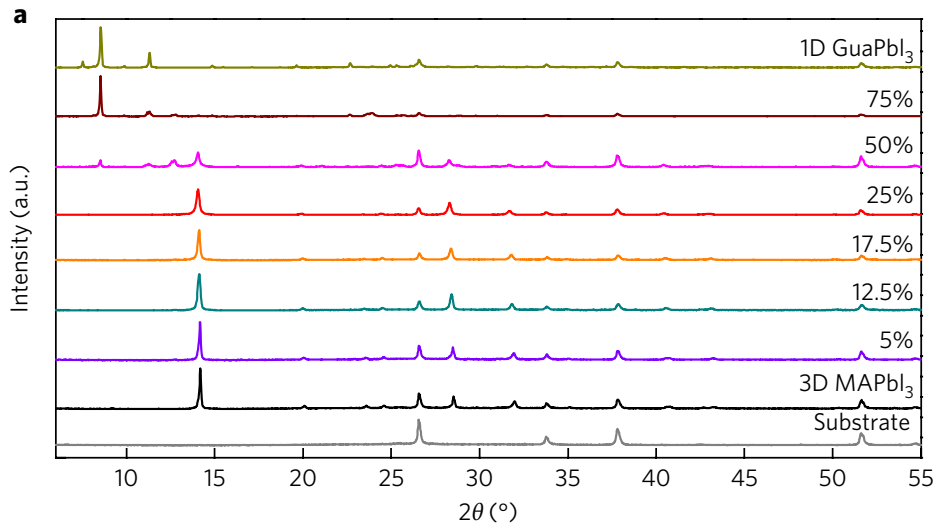

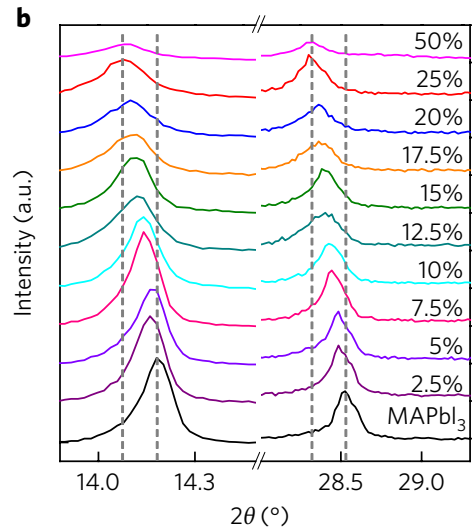
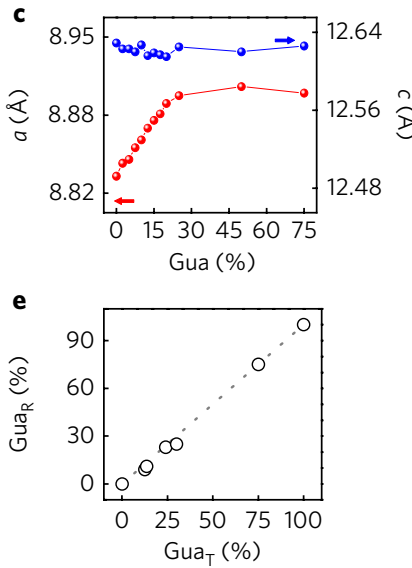

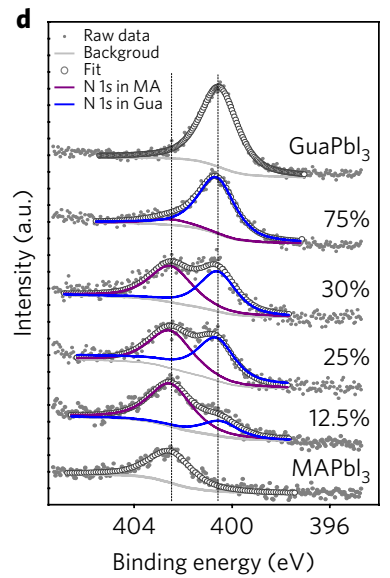

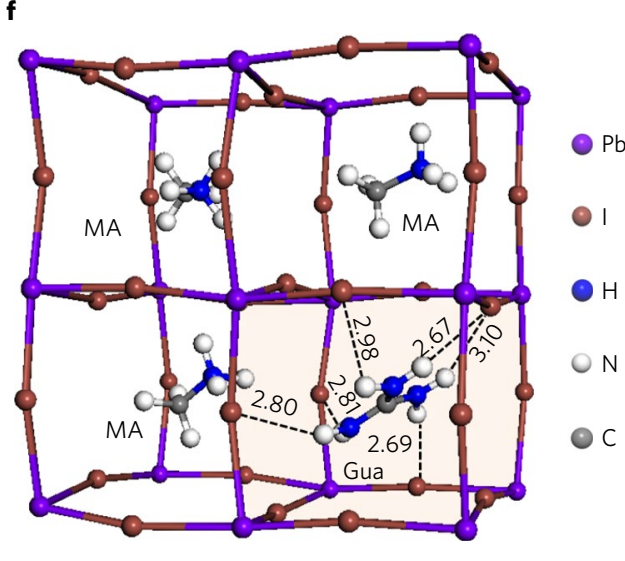

Fig. 1 XRD and XPS characterization of Gua-based perovskites. a, Normalized XRD data for the mixed MA/Gua perovskite films containing different percentages of Gua. b, Magnification of the XRD peaks at (220) (left) and (440) (right) on variation of the Gua content (indicated in per cent). c, Lattice parameter variation ( $a$ and $c$ ) of the $\mathrm{MA}_{1-x} \mathrm{Gua}_{x} \mathrm{Pbl}_{3}$ unit cell obtained from the crystallographic analysis of the experimental XRD patterns. $\mathbf{d}$, XPS spectra of $\mathrm{N} 1 \mathrm{~s}$ for thin films of typical $\mathrm{MA}_{1-x} \mathrm{Gua}_{x} \mathrm{Pbl}_{3}$ perovskite mixtures ( $\left.x=0,12.5,25,30,50,100\right)$ (for clarity, traces are shifted vertically). e, Quantification of the percentage of Gua obtained from the XPS analysis ( $G_{1} a_{R}$ corresponds to the measured values; Gua ${ }_{T}$ corresponds to theoretical values, initially incorporated into the precursor solution). f, Optimized simulated structure of the unit cell of $\mathrm{Ma}_{0.75} \mathrm{Gua}_{0.25} \mathrm{Pbl}_{3}$ including the six $\mathrm{H}$-bond distances with respect to I atoms.

corresponding to the (110) and (220) lattice planes. On increasing the Gua content, a gradual shift of the peaks occurs, as reported for the $\mathrm{FAPbI}_{3} \alpha$-phase after sequential substitution of FA cations for $\mathrm{Cs}^{22}$ or in the recently reported $\mathrm{MA}_{x} \mathrm{EA}_{1-x} \mathrm{PbBr}_{3}$ perovskite (EA= ethylammonium) at increasing EA content ${ }^{23}$. In our case, this phenomenon denotes a gradual expansion of the unit-cell volume, indicating the incorporation of the larger Gua cation by direct substitution of $\mathrm{MA}$, forming a mixed $\mathrm{MA}_{1-x} \mathrm{Gua}_{x} \mathrm{PbI}_{3}$. The lattice parameters, $a$ and $c$, calculated from the experimental XRD patterns, indicate that the $c$ value remains constant for all percentages, $12.625 \AA$, while $a$ gradually changes from $8.838 \AA$ to $8.902 \AA$, remaining invariable for Gua contents larger than $25 \%$ (Fig. 1c). Thus, a distortion of the crystal prevalently affecting the $a$ and $b$ lattice parameters takes place for $x_{\text {Gua }}<0.25$. Due to the mismatch in size with respect to the MA cation, the incorporation of Gua entails local distortions in the crystal, which manifest as a broadening of the peaks. The crystal strains in the structure are evaluated using the Williamson-Hall method (Supplementary Fig. 1). The results demonstrate an increased number of micro-strains and distortions of the $\mathrm{MAPbI}_{3}$ lattice at larger percentages of Gua, supporting its inclusion in the crystal. However, further increments do not incorporate into the crystalline network and lead to a phase-separated $1 \mathrm{D} \mathrm{GuaPbI}$, as suggested by the XRD. It is worth noting that these results are in contradiction to those of previous reports, where Gua has been proposed as a passivating agent that does not incorporate into the perovskite structure due to its larger size ${ }^{24}$. Nevertheless, significant variations in the preparation method could account for the different crystallization dynamics. Here, we incorporate Gua within the precursor solution, ensuring the desired stoichiometry during crystallization.

The relative composition and chemical environment of the constituents in the perovskite materials have been analysed by X-ray photoelectron spectroscopy (XPS). Measurements performed for the pure MA and Gua phases and four representative mixtures $\left(x_{\mathrm{Gua}}=12.5 \%, 25 \% 30 \%\right.$ and $\left.50 \%\right)$ reveal the formation of $\mathrm{Pb}-\mathrm{I}$ bonds and a relative $\mathrm{Pb} / \mathrm{I}$ ratio of $\sim 3.0$, consistent with the theoretical stoichiometry present in the $3 \mathrm{D} \mathrm{MA}_{1-x} \mathrm{Gua}_{x} \mathrm{PbI}_{3}$ and $1 \mathrm{D} \mathrm{GuaPbI}$ structures (Supplementary Fig. 2a). In addition, the photoemission spectra of the $\mathrm{N} 1 s$ region reveals the two peaks attributed to the $\mathrm{NH}_{3}{ }^{+}$group in $\mathrm{MA}(402.3 \mathrm{eV})$ and the $\mathrm{NH}_{2}{ }^{+}$group in $\mathrm{Gua}(400.3 \mathrm{eV})$, which changes gradually in intensity on increasing the Gua content (Fig. 1d). The initial experimental MA/Gua ratio is preserved within the final structure, as presented in Fig. 1e (corroborated by NMR measurements, Supplementary Fig. 2b,c), which is particularly valuable in the determination of the crystalline structure.

To analyse the role of Gua in the crystalline material and examine the stability of the $\mathrm{MA}_{1-x} \mathrm{Gua}_{x} \mathrm{PbI}_{3}$ structures, density functional theory with generalized gradient approximation (DFT-GGA) calculations using the Perdew-Burke-Ernzerhof (PBE) exchange 

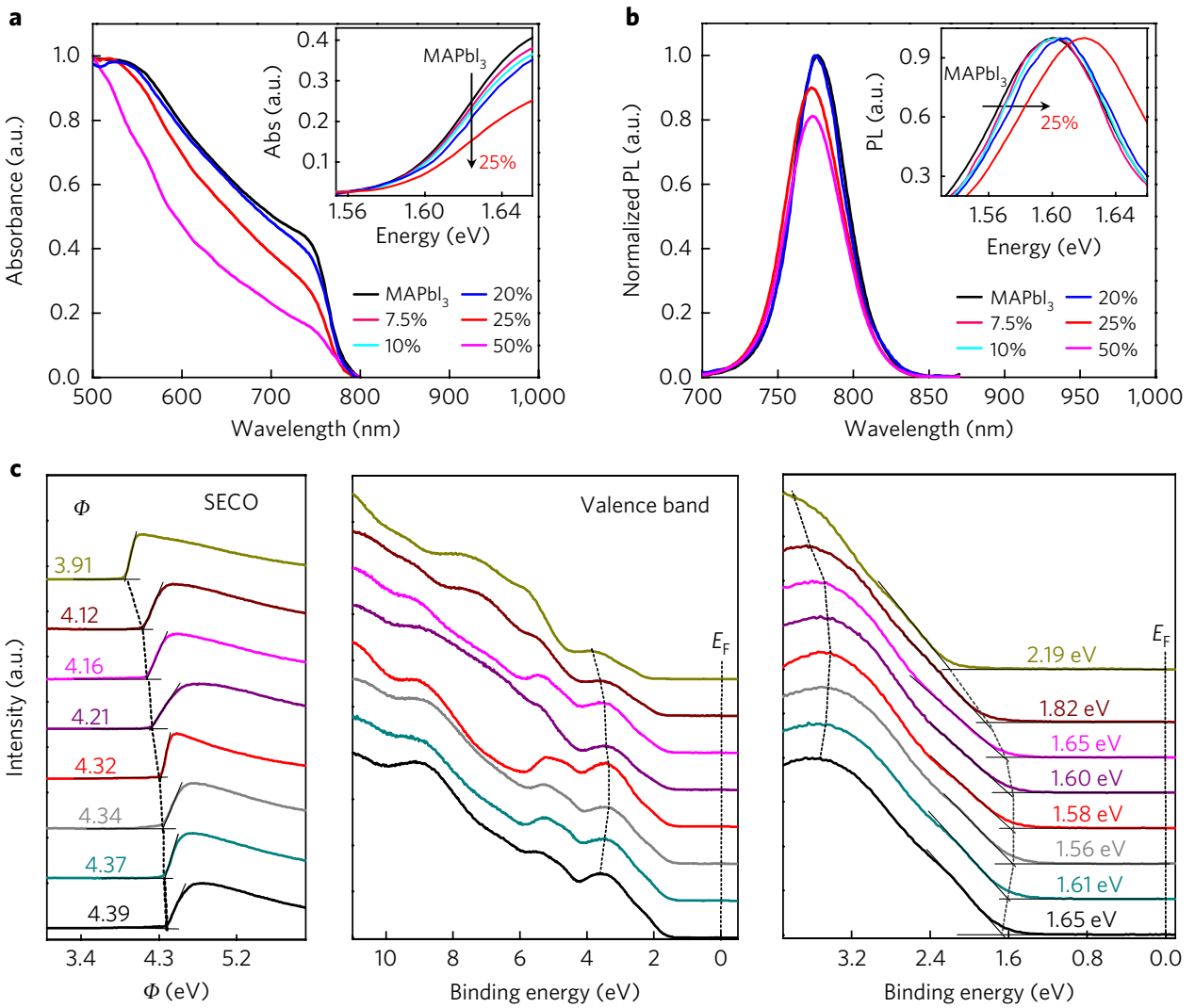

Fig. 2 | UPS and optical characterization. a, Ultraviolet-visible-near-infrared absorption spectra of typical $\mathrm{MA}_{1-x} \mathrm{Gua}_{x} \mathrm{Pbl}_{3}$ films containing $x \leq 0.5$ shown in Fig. 1. Inset: magnification of the band edge for mixed films containing $x \leq 0.25$ of Gua. b, Photoluminescence (PL) spectra of the perovskite films shown in a. c, Secondary-electron cutoffs for workfunction determination (left), and wide (centre) and narrow (right) binding energy range valence spectra of the samples with different Gua/MA ratios.

correlation functional were conducted. An estimation of the formation enthalpies at zero temperature, $\Delta H_{\mathrm{f}}$, for the pure MA and Gua phases, as well as for that containing $x_{\text {Gua }}=0.25$ was performed (Supplementary Note 1). A remarkable difference for the three perovskite structures was found, with a clear tendency when increasing the Gua content: $\Delta H_{\mathrm{f}(\mathrm{MA} \text { pure })}=-0.149 \mathrm{eV} \gg \Delta H_{\mathrm{f}(\mathrm{MA0} .75 \text { Gua } 0.25)}$ $=-1.484 \mathrm{eV}>\Delta H_{\mathrm{f}(\text { Gua pure })}=-2.045 \mathrm{eV}$. Compared with the pure $\mathrm{MAPbI}_{3}$ phase, which exhibits a value close to zero, consistent with previous reports ${ }^{25,26}, 1 \mathrm{D} \mathrm{GuaPbI}_{3}$ points to enhanced stability, as expected for $\mathrm{LDP}^{27}$. However, a striking result appears for the mixed $\mathrm{Ma}_{0.75} \mathrm{Gua}_{0.25} \mathrm{PbI}_{3}$ perovskite, which also exhibits a highly negative formation enthalpy, close to that of the $1 \mathrm{D} \mathrm{GuaPbI}$, while maintaining its 3D structure. Note that such a behaviour has important implications for the performance of the solar cell, as the substitution of Gua for $1 / 4$ of MA in the $\mathrm{MAPbI}_{3}$ structure results in a significant increase of the system stability. Besides, a phase separation of the $\mathrm{MA}_{0.75} \mathrm{Gua}_{0.25} \mathrm{PbI}_{3}$ into the pure MA and Gua systems was found to be thermodynamically less favoured $\left(\Delta H_{\mathrm{f}=}-0.622 \mathrm{eV}\right)$, and it is foreseen that it does not occur at a lower percentage of Gua. Only at additions higher than $25 \%$, both $\mathrm{Ma}_{0.75} \mathrm{Gua}_{0.25} \mathrm{PbI}_{3}$ and $\mathrm{GuaPbI}_{3}$ crystalline phases appear, given the impossibility of the $3 \mathrm{D}$ octahedral $\left[\mathrm{PbI}_{6}\right]$ network to accommodate additional Gua cations. Figure if shows the optimized unit cell for $\mathrm{Ma}_{0.75} \mathrm{Gua}_{0.25} \mathrm{PbI}_{3}$, where the main $\mathrm{H}-\mathrm{I}$ interactions with the Gua cation can be identified. Note that the formation of hydrogen bonds plays a key role in the structural stabilization. Compared with the 1-2 $\mathrm{H}$ bonds per MA molecule $^{28}$, the introduction of Gua increases the number of interactions to six $\mathrm{H}$ bonds while reducing the $\mathrm{H}-\mathrm{I}$ distance, which seems to be the ultimate cause for the superior $\mathrm{Ma}_{0.75} \mathrm{Gua}_{0.25} \mathrm{PbI}_{3}$ stability. Importantly, our results suggest that the crystal distortions produced by the larger cation could be compensated at the expense of the neighbouring cavities where the small MA cation localizes, preserving its $3 \mathrm{D}$ structure.

\section{Optical and electronic properties}

The effect of the lattice expansion on the optoelectronic properties was also analysed by DFT-GGA calculations. The electronic band structures and density of states for $\mathrm{MAPbI}_{3}$ and two representative mixtures $(12.5 \%$ and $25 \%$ of Gua content) show no appreciable difference, with I $5 p$ states mainly contributing to the top valence band and $\mathrm{Pb} 6 p$ states dominating the lowest conduction band (Supplementary Fig. 3). Nevertheless, there is a small but gradual increase in the bandgap energy with the insertion of Gua, showing values equal to $1.77 \mathrm{eV}, 1.79 \mathrm{eV}$ and $1.82 \mathrm{eV}$ for $\mathrm{MAPbI}_{3}, 12.5 \%$ and $25 \%$ of Gua, respectively. The direct character of the $\mathrm{MAPbI}_{3}$ bandgap is also conserved in the two mixtures, with the minimum bandgap occurring at the $\Gamma$ symmetry point. Moreover, the three samples display a similar dispersion of the valence and conduction bands, which ensures low carrier effective masses.

Consequently, the incorporation of Gua preserves the optical properties of the material. Figure 2a shows the normalized absorption spectra for four typical $\mathrm{Ma}_{1-x} \mathrm{Gua}_{x} \mathrm{PbI}_{3}$ thin films with $x_{\mathrm{Gua}}<0.5$. In agreement with the theoretical calculations, no significant changes in the shape of the spectrum are observed for mixtures containing $x_{\text {Gua }}<0.2$, except a tiny but continuous shift in the absorption band edge, as illustrated in the inset of Fig. 2a. Yet, $x_{\mathrm{Gua}}>0.2$ produces an appreciable lowering of the absorption capacity along with a blueshift of the band edge $(\sim 0.02 \mathrm{eV})$, indicative of a widening of the bandgap. This can be related to the different organic-inorganic interactions mediated by the new hydrogen bonds, which lead to 

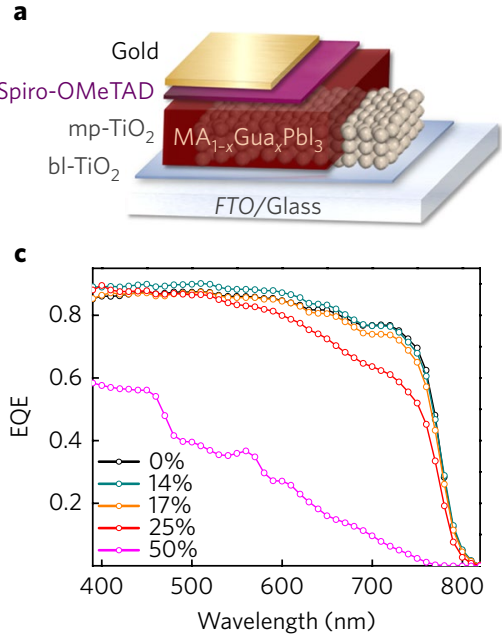
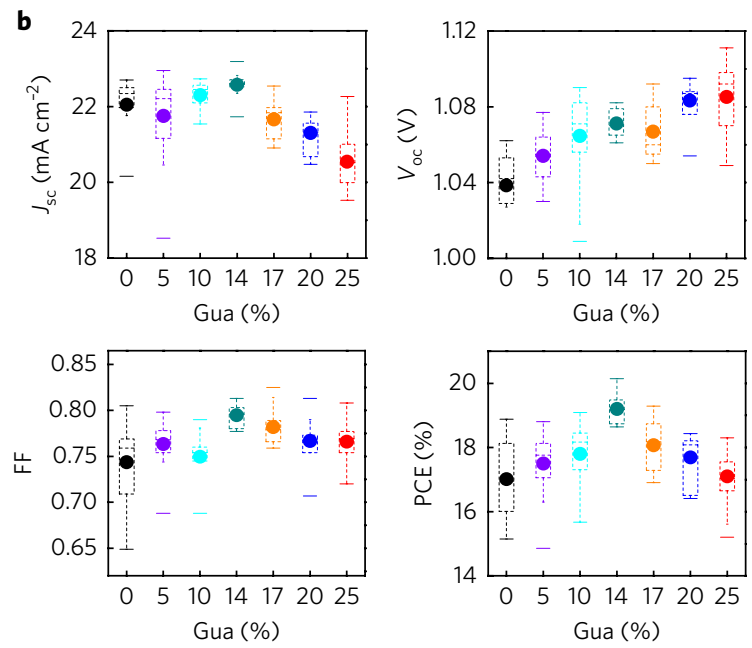

Fig. 3 | Device architecture and photovoltaic performance. a, Device architecture of the perovskite solar cells incorporating Gua cations (glass/FTO/ $\mathrm{c}-\mathrm{TiO}_{2} / \mathrm{mp}-\mathrm{TiO}_{2} /$ perovskite/spiro-OMeTAD/Au). b. Statistical data for $J_{\mathrm{sc}} \mathrm{V}_{\mathrm{oc}} \mathrm{FF}$ and PCE obtained from more than 120 cells (at least 17 cells per condition) prepared with $\mathrm{MA}_{1-x} \mathrm{Gua}_{x} \mathrm{Pbl}_{3}$ as a function of $x$. The top bar shows the maximum value, the bottom bar shows the minimum value, the circle shows the mean value and the dashed rectangle shows the region containing $25-75 \%$ of the data, obtained for each condition. $\mathbf{c}$, EQE spectra of mixed $\mathrm{MA}_{1-x} \mathrm{Gua}_{x} \mathrm{Pbl}_{3}$ $(x=0.14,0.17$ and 0.25$)$ and that incorporating $50 \%$ Gua. The result for $\mathrm{MAPbl}_{3}(0 \%)$ is also included as a reference.

local distortions at the Gua neighbouring positions, and expand the crystal unit. Results for higher Gua percentages are also reported in Supplementary Fig. 4a. A sharp band edge at $\sim 500 \mathrm{~nm}$, with a remarkable excitonic peak at $390 \mathrm{~nm}$ appears for pure $1 \mathrm{D} \mathrm{GuaPbI}_{3}$ (ref. ${ }^{21}$ ). This is also detected for $x_{\mathrm{Gua}}>0.5$, which, together with the absorption band edge of $\mathrm{Ma}_{0.75} \mathrm{Gua}_{0.25} \mathrm{PbI}_{3}$ at $\sim 775 \mathrm{~nm}$, confirms the preservation of both individual phases. Accordingly, the photoluminescence (PL) spectra shown in Fig. 2b (Supplementary Fig. 4b-d) manifest a gradual shift towards higher energy for samples with $x_{\text {Gua }}<0.2$ (see inset to Fig. 2b), which becomes more apparent for $x_{\text {Gua }}=0.25$, and is preserved for larger Gua amounts, consistent with the results observed in Fig. 2a. These findings provide compelling evidence that the substitution of Gua for MA efficiently stabilizes a $3 \mathrm{D}$ perovskite phase while retaining extended and efficient absorption similar to $\mathrm{MAPbI}_{3}$.

In addition, the electronic structure was also investigated by ultraviolet photoelectron spectroscopy (UPS). The results reveal a slight gradual shift of the valence band onset (by $0.04-0.09 \mathrm{eV}$ ) towards the Fermi level from $\mathrm{MAPbI}_{3}$ to $\mathrm{MA}_{1-x} \mathrm{Gua}_{x} \mathrm{PbI}_{3}$ samples containing up to $25 \%$ Gua, while retaining the main valence band features of $\mathrm{MAPbI}_{3}$, as depicted in Fig. 2c. Concomitantly, the ionization energy becomes slightly reduced (by up to $0.14 \mathrm{eV}$ ). However, larger amounts of Gua $(>25 \%)$ result in a shift of the valence band onset away from the Fermi level. A solid change in the valence band features, increasingly resembling those corresponding to pure $\mathrm{GuaPbI}_{3}$ (binding energy region between $5.5 \mathrm{eV}$ and $8.7 \mathrm{eV}$ ), suggests the coexistence of $\mathrm{GuaPbI}_{3}$ at the surface above $25 \%$ Gua content, which is corroborated also by scanning electron microscopy (SEM; as discussed below). As GuaPbI ${ }_{3}$ contains an increased number of $\mathrm{Pb}^{0}$ related surface states, which tend to pin the Fermi level close to the conduction band minimum ${ }^{29}$ (Supplementary Fig. 2a), the wider gap of $\mathrm{GuaPbI}_{3}$ as compared with $\mathrm{MAPbI}_{3}$ results in the shift of the valence band onset towards higher binding energy for $x_{\mathrm{Gua}}>0.3$.

\section{Photovoltaic performance}

To demonstrate the applicability of Gua-containing perovskites, we embodied the $\mathrm{Ma}_{1-x} \mathrm{Gua}_{x} \mathrm{PbI}_{3}(0<x<0.25)$ into solar cells sandwiched in between the mesoporous (mp)- $\mathrm{TiO}_{2}$, used as the electron-transporting material, and $2,2^{\prime}, 7,7^{\prime}$-tetrakis $\left(N, N^{\prime}\right.$-di- $p$-methoxyphenylamine)-9,9'-spirobifluorene (spiro-OMeTAD), as the hole-transporting material (Fig. 3a and Supplementary Fig. 5). The average photovoltaic parameters obtained from the cells are shown in Fig. $3 \mathrm{~b}$ and listed in Table 1, which also includes the champion cells obtained for each condition (see also Supplementary Table 1). The short-circuit current density $\left(J_{s c}\right)$ hardly changes for Gua fractions up to $20 \%$, but gradually lowers for increased Gua contents, with $14 \%$ of Gua outstanding among the others. This behaviour can be related to the decreased absorption detected for $x_{\text {Gua }}>0.2$, as supported by the external quantum efficiency (EQE) spectra shown in Fig. 3c (photographs of the as-prepared $\mathrm{MA}_{1-x} \mathrm{Gua}_{x} \mathrm{PbI}_{3}$ solar cells are provided in Supplementary Fig. 6). In addition, the average open-circuit voltage $\left(V_{\mathrm{oc}}\right)$ increases progressively with the Gua incorporation, from $1.04 \pm 0.02 \mathrm{~V}$ for $x=0$ to $1.085 \pm 0.02 \mathrm{~V}$ for $x=0.25$ (see Table 1 ), as expected from the optical bandgap observations. Notably, the fill factor (FF) remains almost unaltered, with high average values over 0.75 (and maxima close to 0.80). As a result, the PCE of the prepared cells increases from $17 \pm 1 \%$ for $\mathrm{MAPbI}_{3}$ to $19.2 \pm 0.4 \%$ for the Gua/MA mixed system containing $14 \%$ Gua, with a champion cell efficiency of $20.15 \%$. Moreover, even for perovskites containing 25\% Gua, the photovoltaic performance remains similar to that of the $\mathrm{MAPbI}_{3}$, with an average PCE of $17.1 \pm 0.8 \%$ and a record efficiency of $18.30 \%$ (Supplementary Fig. 7). The current density versus voltage $(J-V)$ curve and EQE obtained for the champion device are presented in Fig. 4a,b, respectively. The EQE demonstrates high photon-to-current conversion over $80 \%$ throughout the entire ultraviolet-visible spectrum, leading to an integrated current density of $22.09 \mathrm{~mA} \mathrm{~cm}^{-2}$. $J-V$ hysteresis measurements are reported in Supplementary Fig. 8. We also analysed the charge dynamics by electro-chemical impedance spectroscopy (Supplementary Figs. 9 and 10), which suggest an increased recombination resistance and longer electron lifetime with Gua incorporation, possibly related to a more efficient charge extraction and better crystal formation.

\section{Device stability and phase segregation analysis}

The device stability has been tested under AM1.5G Sun illumination at maximum power point (MPP) tracking, for more than 1,000 hours, at $60^{\circ} \mathrm{C}$ and under an Ar atmosphere. The results (Fig. 4d) reveal an enhanced stability for higher Gua contents, providing evidence of the beneficial effect on the material stability, as predicted by the simulations. Despite the initial decrease, recently 


\begin{tabular}{|c|c|c|c|c|}
\hline $\begin{array}{l}\text { Gua } \\
(\%)\end{array}$ & $J_{\mathrm{sc}}\left(\mathrm{mA} \mathrm{cm}{ }^{-2}\right)$ & $V_{\text {oc }}(\mathrm{V})$ & FF & PCE (\%) \\
\hline 0 & $\begin{array}{l}22.50 \\
(22.0 \pm 0.8)\end{array}$ & $\begin{array}{l}1.050 \\
(1.04 \pm 0.02)\end{array}$ & $\begin{array}{l}0.80 \\
(0.74 \pm 0.04)\end{array}$ & $\begin{array}{l}18.88 \\
(17 \pm 1)\end{array}$ \\
\hline 5.00 & $22.49(22 \pm 1)$ & $\begin{array}{l}1.070 \\
(1.054 \pm 0.01)\end{array}$ & $\begin{array}{l}0.782 \\
(0.76 \pm 0.02)\end{array}$ & $18.81(17 \pm 1)$ \\
\hline 10.0 & $\begin{array}{l}22.53 \\
(22.3 \pm 0.3)\end{array}$ & $\begin{array}{l}1.090 \\
(1.07 \pm 0.02)\end{array}$ & $\begin{array}{l}0.777 \\
(0.75 \pm 0.03)\end{array}$ & $\begin{array}{l}19.09 \\
(17.8 \pm 0.9)\end{array}$ \\
\hline 14.0 & $\begin{array}{l}23.19 \\
(22.8 \pm 0.3)\end{array}$ & $\begin{array}{l}1.082 \\
(1.07 \pm 0.01)\end{array}$ & $\begin{array}{l}0.803 \\
(0.79 \pm 0.01)\end{array}$ & $\begin{array}{l}20.15 \\
(19.2 \pm 0.4)\end{array}$ \\
\hline 17.0 & $\begin{array}{l}22.54 \\
(21.7 \pm 0.5)\end{array}$ & $\begin{array}{l}1.091 \\
(1.08 \pm 0.02)\end{array}$ & $\begin{array}{l}0.785 \\
(0.78 \pm 0.02)\end{array}$ & $\begin{array}{l}19.29 \\
(18.1 \pm 0.7)\end{array}$ \\
\hline 20.0 & $\begin{array}{l}21.31 \\
(21.3 \pm 0.5)\end{array}$ & $\begin{array}{l}1.095 \\
(1.08 \pm 0.01)\end{array}$ & $\begin{array}{l}0.790 \\
(0.77 \pm 0.03)\end{array}$ & $\begin{array}{l}18.43 \\
(17.7 \pm 0.8)\end{array}$ \\
\hline 25.0 & $\begin{array}{l}20.85 \\
(20.6 \pm 0.7)\end{array}$ & $\begin{array}{l}1.107 \\
(1.09 \pm 0.02)\end{array}$ & $\begin{array}{l}0.790 \\
(0.77 \pm 0.02)\end{array}$ & $\begin{array}{l}18.30 \\
(17.1 \pm 0.8)\end{array}$ \\
\hline
\end{tabular}

Photovoltaic parameters obtained for the best performing cells containing MA/Gua perovskite systems measured under AM1.5G Sun illumination. In brackets are indicated the averaged values for each condition (scan rate: $0.01 \mathrm{~V} \mathrm{~s}^{-1}$; stabilization time: $5 \mathrm{~s}$ ).

associated with the inter-penetration of spiro-OMeTAD and gold electrode ${ }^{30}$, a gradual stabilization and recuperation of the performance is observed, which is reproducible (Supplementary Fig. 11a) and clearly correlated with the Gua content. Alternatively, we have performed an initial light stress test at $85^{\circ} \mathrm{C}$ using polytriarylamine polymer, a more stable hole-transporting material, under $300 \mathrm{~h}$ of illumination, which further confirms the enhanced stability compared with $\mathrm{MAPbI}_{3}$, for the mixed MA/Gua perovskite (Supplementary Fig. 11b).

To gain insight into the phase stability and micro-structure of the mixed MA/Gua system, we analysed the film morphology and phase segregation using SEM and combined micro-Raman and micro-photoluminescence (micro-PL) spectroscopy. Figure $5 \mathrm{a}-\mathrm{d}$ shows the SEM images obtained for four representative compositions, including the pure $\mathrm{MAPbI}_{3}$ and $\mathrm{GuaPbI}_{3}$ phases, as well as samples containing Gua below and above $25 \%$, at which the phase segregation may occur. As presented in the figure, the $\mathrm{MAPbI}_{3}$ film has a grainy-like morphology with large crystal domains of around $1 \mu \mathrm{m}$ diameter (see orange line). On the contrary, $\mathrm{GuaPbI}_{3}$ presents an elongated rod-like morphology with crystals of several micrometres in length, typical for the 1D aggregation. Notably, samples containing $x_{\text {Gua }}<0.25$ do not show any similar phase, resembling the $\mathrm{MAPbI}_{3}$ but with a considerably increased size of the crystal domains, as emphasized by the orange line in the picture. A relation between the amount of Gua and the size of the crystal domains is also observed for all samples, in which the domains remain like islands of several micrometres within the capping layer (Supplementary Figs. 12 and 13). The 1D-like morphology is detected only for samples containing higher amounts of Gua $(>25 \%)$, as also appreciated in Fig. 5, at which phase segregation occurs.

These results are confirmed by the micro-Raman and micro-PL analysis (Fig. 5e,f). Averaged over a diffraction-limited spot size of $300 \mathrm{~nm}$, this technique can provide further information about local heterogeneities within the film ${ }^{31}$. The emission of four different samples was investigated, with a particular focus on the distribution of the PL peak position. The latter is intimately linked to the material bandgap, which changes with composition ${ }^{9}$ and/or local disor$\mathrm{der}^{32}$ over microscopic areas of the samples. Fig. 5e shows the map of the PL peak position over $6 \times 6 \mu \mathrm{m}^{2}$ regions. In the wavelength emission range between $710 \mathrm{~nm}$ and $780 \mathrm{~nm}$, no appreciable change in the PL peak is observed for $x_{\text {Gua }}<0.25$, remaining around $770 \mathrm{~nm}$, as for the pure $\mathrm{MAPbI}_{3}$ (Supplementary Fig. 14). On the contrary, the addition of $75 \%$ Gua leads to the appearance of a contrasted PL map with regions extending up to a few micrometres, with a remarkable PL shift down to $710 \mathrm{~nm}$. This behaviour indicates a phase segregation in the material, resulting in a distribution of different bandgaps across the film. On the same areas, micro-Raman spectra were recorded (Fig. 5f). For Gua contents up to 25\%, the Raman spectra closely resemble that for the pure $\mathrm{MAPbI}_{3}$. Characteristic broad peaks in the region below $200 \mathrm{~cm}^{-1}$ are observed related to the $\mathrm{Pb}-\mathrm{I}$ stretching and bending modes ${ }^{33,34}$, along with a broad feature around $250 \mathrm{~cm}^{-1}$ related to the vibrations of the organic cation. Remarkably, as recently observed with FA incorporation to the $\mathrm{MAPbI}_{3}$, a gradual shift of the peak to lower wavenumber appears,
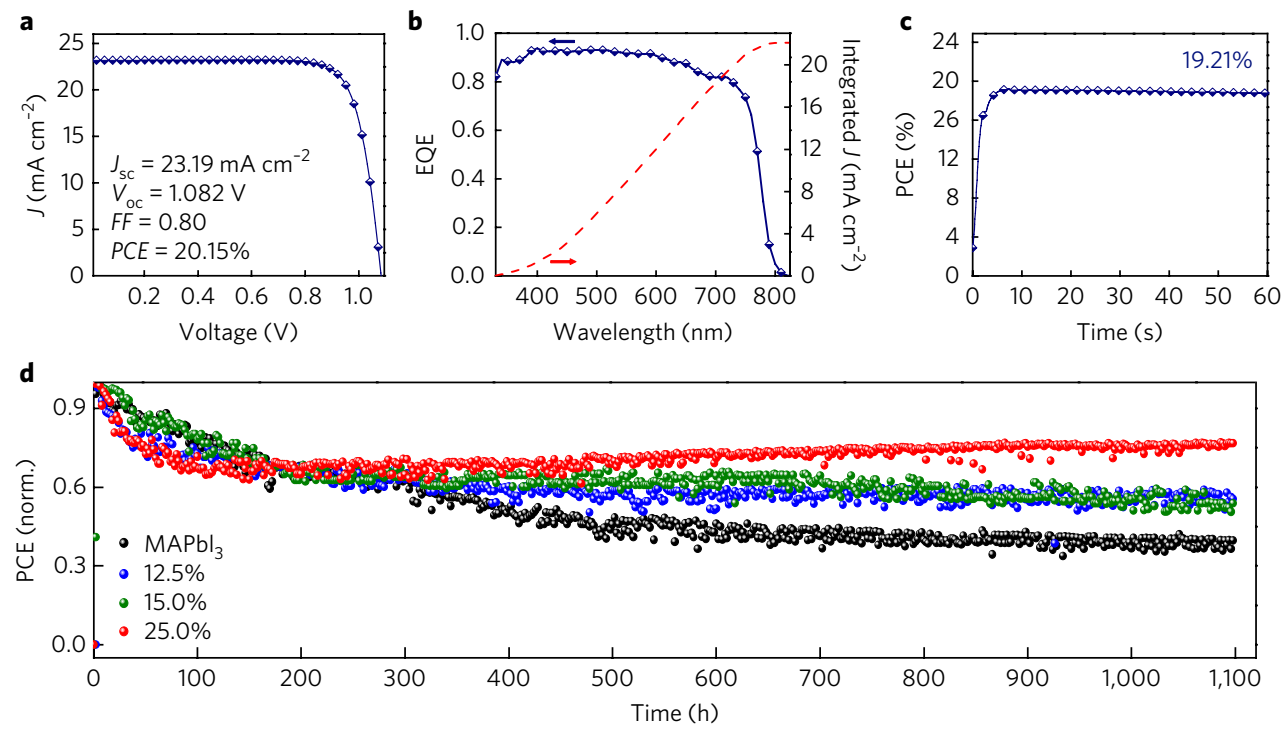

Fig. 4 | Champion device and thermal stability test. a,b, J-V curve (a) and EQE spectrum (b) of the champion cell prepared with mixed MA/Gua perovskite containing $14 \%$ Gua. c, MPP tracking under 1 Sun AM1.5G illumination measured in air, for a typical high-efficiency cell $\left(\mathrm{MA}_{1-x} \mathrm{Gua}_{x} \mathrm{Pbl}_{3}\right.$ $(x=0.14))$. $\mathbf{d}$, Thermal stability test of $\mathrm{MA}_{1-x} \mathrm{Gua}_{x} \mathrm{Pbl}_{3}$ perovskite solar cells $(x=0,0.125,0.15,0.25)$ at $60^{\circ} \mathrm{C}$ under continuous light illumination and MPP tracking in an argon atmosphere. The initial PCE for each cell was $18.77 \%, 18.97 \%, 18.11 \%$, and $17.14 \%$, respectively. 


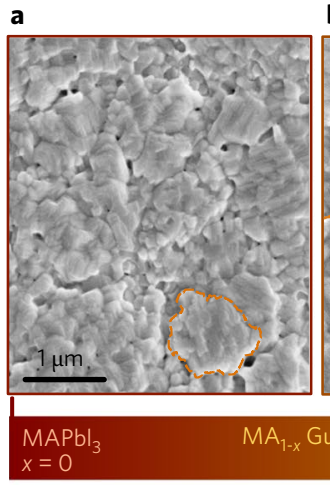

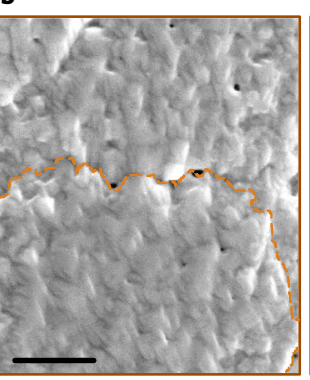

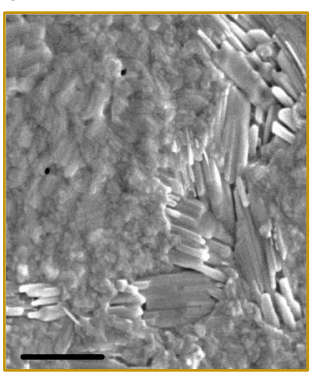

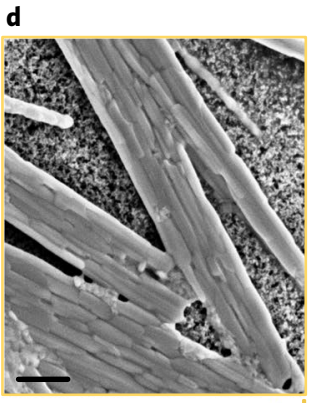

$\mathrm{GuaPbl}_{3}$

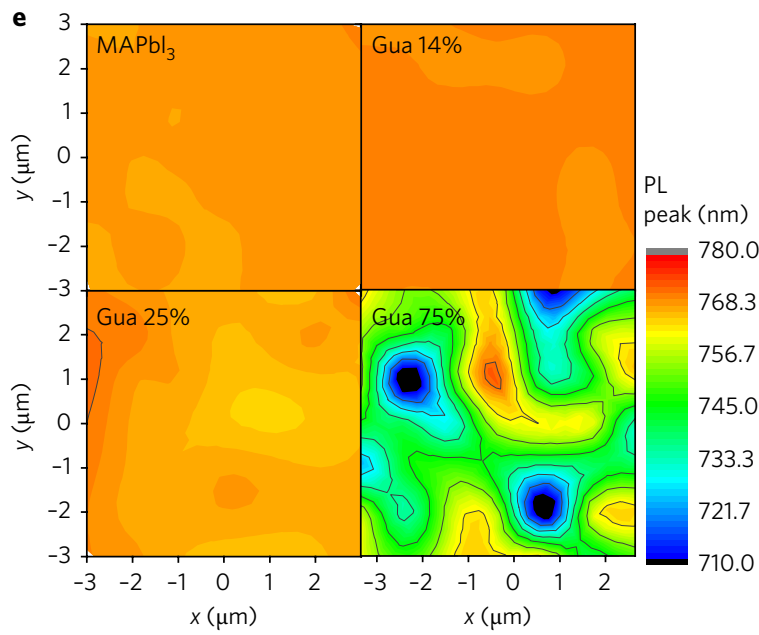

f

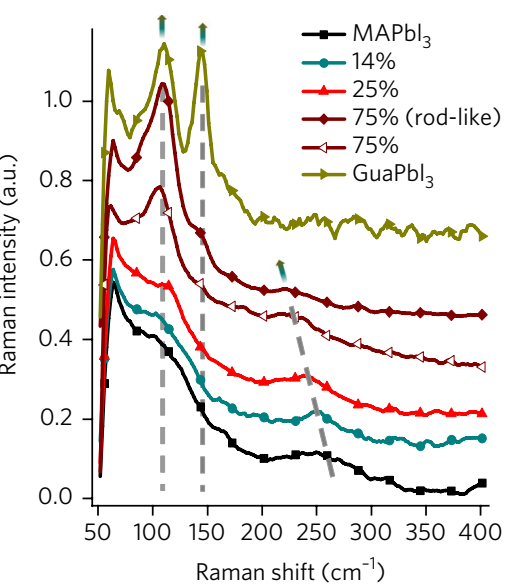

Fig. 5 | Morphology and phase segregation analysis. a-d, Top-surface SEM images of four representative samples, including pure $\mathrm{MAPb}_{3}(\mathbf{a})$, mixed $\mathrm{MA}_{1-x} \mathrm{Gua}_{x} \mathrm{Pbl}_{3}$ with $x=0.14(\mathbf{b})$, with $x=0.30(\mathbf{c})$, and pure GuaPbl${ }_{3}(\mathbf{d})$. Scale bars, $1 \mu \mathrm{m}$. e,f, Micro-PL wavelength peak shift map (e) and micro-Raman spectra (f) of the perovskite surface obtained for Gua contents equal to 0, 15, 25 and 75\%. The traces are shifted vertically for clarity.

suggesting a shrinking of the mode due to the incorporation of the distinct Gua cation in the unit cell. For Gua content above 25\%, we could identify a position-dependent signal that changes in line with the PL variation. Some regions show a similar spectrum as for the lower Gua content, while on the regions presenting the 'rod-like' morphology, a redistribution of the relative peak intensity happens with a dominant peak at $120 \mathrm{~cm}^{-1}$ and an additional peak at $135 \mathrm{~cm}^{-1}$, both with a reduced broadening of the signal. This peak shift is usually attributed to isolated $\mathrm{Pb}-\mathrm{I}$ planes, similarly to the case of $\mathrm{PbI}_{2}$ intercalated with large organic molecules ${ }^{35}$, which suggest the formation of a LDP as for the pure $\mathrm{GuaPbI}_{3}$ arranging into the $1 \mathrm{D}$ phase. As corroborated in the figure, $\mathrm{GuaPbI}_{3}$ shows apparent peaks at $120 \mathrm{~cm}^{-1}$ and $135 \mathrm{~cm}^{-1}$ as previously asserted for the $75 \%$ sample. These results further confirm the phase homogeneity of the mixed $\mathrm{MA}_{1-x} \mathrm{Gua}_{x} \mathrm{PbI}_{3}$ for Gua $<25 \%$, which, on the contrary, reveals a severe phase segregation into a 3D/1D mixture for larger Gua amounts, while retaining the individual features of the single constituents. No visible degradation signs are observed during the PL measurements, as shown in Supplementary Fig. 14b.

\section{Conclusions}

We present a perovskite composition based on a combination of Gua/MA cations that exhibits superior photovoltaic performance and material stability compared with $\mathrm{MAPbI}_{3}$. We demonstrate that the incorporation of large Gua cations unexpectedly forms a highly stable 3D crystalline structure, plausibly mediated by the increased number of $\mathrm{H}$ bonds within the inorganic framework. The prepared $\mathrm{MA}_{1-x} \mathrm{Gua}_{x} \mathrm{PbI}_{3}$ perovskite preserved the good optoelectronic properties associated with the organic lead halide materials, leading to a high PCE surpassing 20\% for a Gua content of $14 \%$. Our results emphasize the versatility of organicinorganic lead halide perovskites, and invite further exploration of organic cations including those that are beyond the limit of the tolerance factor.

\section{Methods}

Solar cell fabrication. Perovskite solar cells were fabricated on F-doped $\mathrm{SnO}_{2}$ (NSG10) substrates previously cleaned by a sequential sonication treatment in a 2\% Hellmanex solution, acetone and isopropanol, followed by ultravioletozone treatment for $15 \mathrm{~min}$. A compact blocking layer of $\mathrm{TiO}_{2}\left(\mathrm{bl}-\mathrm{TiO}_{2}, 30 \mathrm{~nm}\right.$ in thickness) was then deposited onto the fluorine-doped tin oxide (FTO) glass substrate by spray pyrolysis, using a titanium diisopropoxide bis(acetylacetonate) solution in ethanol $(60 \% \mathrm{v} / \mathrm{v})$, and then sintered at $450^{\circ} \mathrm{C}$ for $30 \mathrm{~min}$. A $200-\mathrm{nm}-$ thick layer of mesoporous $\mathrm{TiO}_{2}\left(\mathrm{mp}-\mathrm{TiO}_{2}, 30 \mathrm{NR}-\mathrm{D}\right.$ titania paste from Dyesol) was prepared by spin-coating a diluted $\mathrm{TiO}_{2}$ dispersion in ethanol, ratio $1: 8$ by weight, at 2,000 r.p.m. for $15 \mathrm{~s}$ followed by a sintering step at $500^{\circ} \mathrm{C}$ for $30 \mathrm{~min}$. Afterwards, the substrates were lithium-treated by spin-coating $40 \mu \mathrm{l}$ of tris (bis (trifluoromethylsulfonyl)imide) (Li-TFS) $\left(14.67 \mathrm{~g} \mathrm{ml}^{-1}\right.$ in acetonitrile) onto the mesoporous layer, followed by a sintering step at $500^{\circ} \mathrm{C}$ for $30 \mathrm{~min}$. Stoichiometric precursor solutions were prepared by mixing MAI, GuaI (Dyesol) and $\mathrm{PbI}_{2}(\mathrm{TCI})$ in $N, N^{\prime}$-dimethylsulfoxide (DMSO) with MAI/GuaI gradually changing from 1:0 to $0: 1$, while keeping the $\mathrm{PbI}_{2}$ molarity equal to 1.25 . The perovskite layers were then fabricated by using a two-step spin-coating process reported previously ${ }^{36}$ (first step 1,000 r.p.m. for $10 \mathrm{~s}$; second step 4,000 r.p.m for $30 \mathrm{~s}$ ), and $15 \mathrm{~s}$ before the end of the programme, $100 \mu \mathrm{l}$ of chlorobenzene was poured onto the films, and then the substrates were annealed at $100^{\circ} \mathrm{C}$ for $45 \mathrm{~min}$. After this time, spiro-OMeTAD was spin-coated at 4,000 r.p.m., for $30 \mathrm{~s}$ from a chlorobenzene solution $(28.9 \mathrm{mg}$ in $400 \mu \mathrm{l}, 60 \mathrm{mmol}$ ) containing Li-TFSI ( $7.0 \mu \mathrm{l}$ from a $520 \mathrm{mg} \mathrm{ml}^{-1}$ stock solution in 
acetonitrile), TBP $(11.5 \mu \mathrm{l})$ and Co(II)TFSI $\left(10 \mathrm{~mol} \%, 8.8 \mu \mathrm{l}\right.$ from a $40 \mathrm{mg} \mathrm{ml}^{-1}$ stock solution) as dopants. Finally, a $70 \mathrm{~nm}$ gold electrode was evaporated.

Thin-film characterization. The XRD patterns of the prepared films were measured using a D8 Advance diffractometer from Bruker (Bragg-Brentano geometry, with an $\mathrm{X}$-ray tube $\mathrm{Cu} \mathrm{K \alpha}, \lambda=1.5406 \AA$ ). The absorption spectra were registered with an ultraviolet-visible-infrared spectrophotometer (PerkinElmer Instrument). Photoluminescence (PL) steady-state measurements were recorded with a spectrophotometer (Gilden Photonics). The micro-PL and micro-Raman measurements were performed on a Renishaw InVia Raman microscope with an L100× objective (spot size of about $300 \mathrm{~nm}$ ). The PL map was recorded using $0.0005 \%$ laser intensity (corresponding to $100 \mu \mathrm{J} \mathrm{cm}^{-2}$ ), using a $532 \mathrm{~nm}$ green laser diode. The spectra were registered in the $710-820 \mathrm{~nm}$ region. Raman spectra were obtained using the same excitation monitoring the $50-250 \mathrm{~cm}^{-1}$ range, particularly sensitive to the $\mathrm{Pb}-\mathrm{I}$ modes. The final data were averaged over 50 accumulations to maximize the signal-to-noise ratio. To prevent sample degradation or thermal effects, the laser power intensity was kept below (excitation density of around $\left.3 \mathrm{~mW} \mathrm{~cm}^{-2}(0.005 \%)\right)$ and the light exposure time per measurement was $0.1 \mathrm{~s}$. Photoelectron spectroscopy measurements were performed in an ultrahighvacuum system (base pressure of $2 \times 10^{-10} \mathrm{mbar}$ ) using a He-discharge ultraviolet source (Omicron) with an excitation energy of $21.2 \mathrm{eV}$ for UPS and an Al Ko X-ray source (excitation energy: $1486.6 \mathrm{eV}$ ) for XPS. The photoelectron spectra were recorded using a Phoibos 100 (Specs) hemispherical energy analyser at a pass energy of $5 \mathrm{eV}$ for the valence band and a pass energy of $20 \mathrm{eV}$ for the core levels. For workfunction determination, the secondary-electron cutoff was recorded by applying a $-10 \mathrm{~V}$ sample bias to clear the analyser workfunction. The reported valence band spectra were background subtracted. The binding energies for all of the photoemission spectra are referenced to the Fermi level. For a comparison of the XPS core-level spectra, the binding energy values were all adjusted to the $\mathrm{C} 1 s$ carbon at lower binding energy that was here referenced to $285 \mathrm{eV}$. A mixed Gaussian/Lorentzian peak shape and a Shirley-type background were employed for XPS peak fitting with the XPS Peak 4.1 software. NMR measurements were performed for samples containing up to $30 \%$ Gua. Mixed $\mathrm{MA}_{1-x} \mathrm{Gua}_{x} \mathrm{PbI}_{3}$ perovskite films were prepared on conductive FTO substrates in the same conditions as for the photovoltaic devices. Once the perovskite layers were crystallized, the solid films were re-dissolved in deuterated DMSO (DMSO-d6) and directly analysed in liquid-state ${ }^{1} \mathrm{H}-\mathrm{NMR} 800 \mathrm{MHz}$ instruments equipped with a $5 \mathrm{~mm}$ triple-channel ${ }^{1} \mathrm{H} /{ }^{13} \mathrm{C} /{ }^{15} \mathrm{~N}$ cryoprobe and a AVII console. The method used was a single pulse with 16 scans, a delay of $5 \mathrm{~s}$ and a pulse length of $7.8 \mu \mathrm{s}$.

Device characterization. The photovoltaic device performance was analysed using a VeraSol LED solar simulator (Newport) producing 1 Sun AM $1.5\left(1,000 \mathrm{~W} \mathrm{~m}^{-2}\right)$ sunlight. Current-voltage curves were measured in air with a potentiostat (Keithley 2604). The light intensity was calibrated with a NREL-certified KG5-filtered Si reference diode. The solar cells were masked with a metal aperture of $0.16 \mathrm{~cm}^{2}$ to define the active area. The cells were measured in air, at room temperature and without encapsulation, at a constant rate $10 \mathrm{mV} \mathrm{s}^{-1}$ for both forward and reverse bias. No special pre-conditioning protocol was used apart from $5 \mathrm{~s}$ of stabilization time before the measurement. No anti-reflective coating was used during the measurement. The stability test was performed in a sealed cell holder flushed with a flow of argon $\left(30 \mathrm{ml} \mathrm{min}^{-1}\right)$ and $I-V$ curves were characterized by an electronic system using a 22-bit delta-sigma analog-to-digital converter, performed every $2 \mathrm{~h}$. For $I-V$ curve measurement, a scan rate of $25 \mathrm{mV} \mathrm{s}^{-1}$ with a step of $5 \mathrm{mV}$ was used, maintaining the temperature of the cells at around $60^{\circ} \mathrm{C}$. Cells were maintained at the maximum power point using a MPP tracking algorithm under $100 \mathrm{~mW} \mathrm{~cm}^{-2}$. A reference $\mathrm{Si}$ photodiode was placed in the holder to verify the stability of the light. EQE was measured with the IQE200B (Oriel) without bias light.

Computational calculations. Geometrical structures of $\mathrm{PbI}_{2}\left(\right.$ ref. ${ }^{37}$ ), GuaI $\left(\right.$ ref. ${ }^{38}$ ), MAI (refs ${ }^{39,40}$ ), tetragonal $\mathrm{MAPbI}_{3} \mathrm{Ma}\left(\right.$ ref. ${ }^{41}$ ) and $1 \mathrm{D} \mathrm{GuaPbI}{ }_{3}$ (ref. ${ }^{21}$ ), from the bibliography, were optimized, without cell optimization. For the different perovskites, $\mathrm{MA}_{1-x} \mathrm{Gua}_{x} \mathrm{PbI}_{3}$, where $x=0,0.25$ and 1 , only the organic material position was optimized, restricting the $\mathrm{Pb}$ and I position to those obtained from the diffractogram. We proceed in this way because the MA geometric arrangement is not known in detail from the information obtained from the diffractograms ${ }^{42,43}$. Periodic DFT-GGA calculations using the PBE exchange correlation functional were performed $^{44}$. Electron-ion interactions were described by ultrasoft pseudopotentials. In the case of the $\mathrm{Pb}$ atom, we used a scalar relativistic pseudopotential. A $4 \times 4 \times 4$ Monkhorst-Pack grid was chosen for sampling the Brillouin zone ${ }^{45}$. Given that these compounds are essentially ionic in nature, electrostatic interactions well described by DFT-GGA are expected to represent the main contribution in the interaction. This is indirectly confirmed by the usually good agreement between experimental and calculated structural parameters for this type of material ${ }^{46}$.

Data availability. The data that support the plots within this paper and other finding of this study are available from the corresponding author upon reasonable request.

Received: 19 April 2017; Accepted: 3 November 2017; Published online: 8 December 2017

\section{References}

1. De Wolf, S. et al. Organometallic halide perovskites: sharp optical absorption edge and its relation to photovoltaic performance. J. Phys. Chem. Lett. 5, 1035-1039 (2014).

2. Stranks, S. D. et al. Electron-hole diffusion lengths exceeding 1 micrometer in an organometal trihalide perovskite absorber. Science 342, 341-344 (2013).

3. Xing, G. et al. Long-range balanced electron- and hole-transport lengths in organic-inorganic $\mathrm{CH}_{3} \mathrm{NH}_{3} \mathrm{PbI}_{3}$. Science 342, 344-347 (2013).

4. Shi, D. et al. Low trap-state density and long carrier diffusion in organo lead trihalide perovskite single crystals. Science 347, 519-522 (2015).

5. Yang., W. S. et al. Iodide management in formamidinium-lead-halide-based perovskite layers for efficient solar cells. Science 356, 1376-1379 (2017).

6. Zhang, Y. et al. Optimization of stable quasi-cubic $\mathrm{FA}_{\mathrm{x}} \mathrm{MA}_{1-\mathrm{x}} \mathrm{PbI}_{3}$ perovskite structure for solar cells with efficiency beyond 20\%. ACS Energy Lett. 2, 802-806 (2017)

7. Bi, D. et al. Efficient luminescent solar cells based on tailored mixed-cation perovskites. Sci. Adv. 2, e1501170 (2016).

8. Conings, B. et al. Intrinsic thermal instability of methylammonium lead trihalide perovskite. Adv. Energy Mater. 5, 1500477 (2015).

9. Gratia, P. et al. Intrinsic halide segregation at nanometer scale determines the high efficiency of mixed cation/mixed halide perovskite solar cells. J. Am. Chem. Soc. 138, 15821-15824 (2016).

10. Hoke, E. T. et al. Reversible photo-induced trap formation in mixed-halide hybrid perovskites for photovoltaics. Chem. Sci. 6, 613-617 (2015).

11. Li, X. et al. Improved performance and stability of perovskite solar cells by crystal crosslinking with alkylphosphonic acid $\omega$-ammonium chlorides. Nat. Chem. 7, 703-711 (2015).

12. You, J. et al. Improved air stability of perovskite solar cells via solutionprocessed metal oxide transport layers. Nat. Nanotech. 11, 75-81 (2016).

13. Kaltenbrunner, M. et al. Flexible high power-per-weight perovskite solar cells with chromium oxide-metal contacts for improved stability in air. Nat. Mater. 14, 1032-1039 (2015).

14. Tsai, H. et al. High-efficiency two-dimensional Ruddlesden-Popper perovskite solar cells. Nature 536, 312-316 (2016)

15. Saliba, M. et al. Cesium-containing triple cation perovskite solar cells: improved stability, reproducibility and high efficiency. Energy Environ. Sci. 9, 1989-1997 (2016).

16. Saliba, M. et al. Incorporation of rubidium cations into perovskite solar cells improves photovoltaic performance. Science 354, 206-209 (2016).

17. Kieslich, G., Sun, S. \& Cheetham, A. K. Solid-state principles applied to organic-inorganic perovskites: new tricks for an old dog. Chem. Sci. 5, 4712-4715 (2014).

18. Goldschmidt, V. M. Die Gesetze der Krystallochemie. Naturwissenschaften 14, 477-485 (1926)

19. Szafrański, M. Investigation of phase instabilities in guanidinium halogenoplumbates(II). Thermochim. Acta 307, 177-183 (1997).

20. Szafrański, M. \& Katrusiak, A. Phase transitions in the layered structure of diguanidinium tetraiodoplumbate. Phys. Rev. B 61, 1026-1035 (2000).

21. Jodlowski, A. D., Yépez, A., Luque, R., Camacho, L. \& de Miguel, G. Benign-by-design solventless mechanochemical synthesis of three-, two-, and one-dimensional hybrid perovskites. Angew. Chem. 55, 14972-14977 (2016).

22. Huang, J., Xu, P., Liu, J. \& You, X.-Z. C. Sequential introduction of cations deriving large-grain $\mathrm{Csx}_{\mathrm{F}} \mathrm{A} 1_{-\mathrm{xP}} \mathrm{bI} 3$ thin film for planar hybrid solar cells: insight into phase-segregation and thermal-healing behavior. Small 13, 1603225 (2016)

23. Gholipour, S. et al. Globularity-selected large molecules for a new generation of multication perovskites. Adv. Mater. 29, 1702005 (2017).

24. Marco, N. D. et al. Guanidinium: a route to enhanced carrier lifetime and open-circuit voltage in hybrid perovskite solar cells. Nano Lett. 16, 1009-1016 (2016).

25. Buin, A., Comin, R., Xu, J., Ip, A. H. \& Sargent, E. H. Halide-dependent electronic structure of organolead perovskite materials. Chem. Mater. 27, 4405-4412 (2015).

26. Nagabhushana, G. P., Shivaramaiah, R. \& Navrotsky, A. Direct calorimetric verification of thermodynamic instability of lead halide hybrid perovskites. Proc. Natl Acad. Sci. USA 113, 7717-7721 (2016).

27. Quan, L. N. et al. Ligand-stabilized reduced-dimensionality perovskites. J. Am. Chem. Soc. 138, 2649-2655 (2016).

28. Lee, J. H., Lee, J.-H., Kong, E.-H. \& Jang, H. M. The nature of hydrogenbonding interaction in the prototypic hybrid halide perovskite, tetragonal $\mathrm{CH}_{3} \mathrm{NH}_{3} \mathrm{PbI}_{3}$. Sci. Rep. 6, 21687 (2016).

29. Zu, F.-S. et al. Impact of white light illumination on the electronic and chemical structures of mixed halide and single crystal perovskites. Adv. Opt. Mater. 5, 1700139 (2017).

30. Domanski, K. et al. Not all that glitters is gold: metal-migration-induced degradation in perovskite solar cells. ACS Nano 10, 6306-6314 (2016).

31. Draguta, S. et al. Spatially non-uniform trap state densities in solutionprocessed hybrid perovskite thin films. J. Phys. Chem. Lett. 7, 715-721 (2016) 
32. Grancini, G. et al. The impact of the crystallization processes on the structural and optical properties of hybrid perovskite films for photovoltaics. J. Phys. Chem. Lett. 5, 3836-3842 (2014).

33. Quarti, C. et al. The Raman spectrum of the $\mathrm{CH}_{3} \mathrm{NH}_{3} \mathrm{PbI}_{3}$ hybrid perovskite: interplay of theory and experiment. J. Phys. Chem. Lett. 5, 279-284 (2014).

34. Grancini, G. et al. $\mathrm{CH}_{3} \mathrm{NH}_{3} \mathrm{PbI}_{3}$ perovskite single crystals: surface photophysics and their interaction with the environment. Chem. Sci. 6, 7305-7310 (2015).

35. Preda, N. et al. Raman and photoluminescence studies on intercalated lead iodide with pyridine and iodine. J. Optoelectron. Adv. Mater. 10, 319-322 (2008).

36. Jeon, N. J. et al. Solvent engineering for high-performance inorganic-organic hybrid perovskite solar cells. Nat. Mater. 13, 897-903 (2014).

37. Wyckoff, R. W. G. Crystal Structures 2nd edn, Vol. 1 239-444 (John Wiley, New York, 1963).

38. Szafranski, M. \& Jarek, M. Origin of spontaneous polarization and reconstructive phase transition in guanidinium iodide. CrystEngComm 15, 4617-4623 (2013)

39. Yamamuro, O. et al. Neutron diffraction and calorimetric studies of methylammonium iodide. Acta Cryst. B48, 329-336 (1992).

40. Ishida, H. et al. EXAFS study on the phase-transition (phase- $\alpha^{\prime}-\delta$ ) in $\mathrm{CH}_{3} \mathrm{NH}_{3}$ I. Z. Naturforsch. A 50, 876-880 (1995).

41. Stoumpos, C. C., Malliakas, C. D. \& Kanatzidis, M. G. Semiconducting tin and lead iodide perovskites with organic cations: phase transitions, high mobilities, and near-infrared photoluminescent properties. Inorg. Chem. 52, 9019-9038 (2013).

42. Motta, C. et al. Revealing the role of organic cations in hybrid halide perovskite $\mathrm{CH}_{3} \mathrm{NH}_{3} \mathrm{PbI}_{3}$. Nat. Commun. 6, 7026-7033 (2015).

43. Ong, K. P., Goh, T. W., Xu, Q. \& Huan, A. Structural evolution in methylammonium lead iodide $\mathrm{CH}_{3} \mathrm{NH}_{3} \mathrm{PbI}_{3}$. J. Phys. Chem. A 119 , 11033-11038 (2015)

44. Perdew, J. P., Burke, K. \& Ernzerhof, M. Generalized gradient approximation made simple. Phys. Rev. Lett. 77, 3865-3868 (1996).

45. Monkhorst, H. J. \& Pack, J. D. Special points for Brillouin-zone integrations Phys. Rev. B 13, 5188-5192 (1976).

46. Lv, H., Gao, H., Yang, Y. \& Liu, L. Density functional theory (DFT) investigation on the structure and electronic properties of the cubic perovskite $\mathrm{PbTiO}_{3}$. Appl. Catal. A 404, 54-58 (2011).

\section{Acknowledgements}

We thank the European Commission H2020-ICT-2014-1, SOLEDLIGHT project, grant agreement no. 643791, the Swiss State Secretariat for Education, Research and Innovation (SERI) and Toyota Motor Europe Technical Center, Advanced Technology division, Home Wei 33, B-1930 Zaventem-Belgium for financial support. G.G. acknowledges the co-funded Marie Skłodowska Curie fellowship, H2020 grant agreement no. 665667, fund number 588072, and the SNSF Ambizione Energy grant SNF project PZENP2_173641. G.M. thanks the Ministry of Economy and Competitiveness for a 'Ramón y Cajal' contract (RYC-2013-12772). G.M. and L.C. acknowledge the Ministry of Economy and Competitiveness for financial support (CTQ2014-56422-P). A.D.J. thanks COSTSTSM-MP1307 for financial support. Work in Berlin was supported by the SFB951 (DFG). We thank E. Oveisi and E. Baudat for the helpful discussions, L. Wen for his support during the XPS measurement and M. Tschumi for providing the instrument required for the stability measurements.

\section{Author contributions}

A.D.J. planned the experiments and prepared and characterized the cells; M.S. performed the impedance measurements and analysis; S.A. supervised M.S.; G.M. performed the XPS, XRD and absorption analysis; G.G. carried out the Raman and PL characterization; C.R.-C. designed the experiments, carried out the SEM analysis and wrote the manuscript; L.C. performed the theoretical simulations; M.R. and N.K. performed UPS and XPS analysis; C.R.-C., G.M., L.C. and M.K.N. conceived the idea, and supervised the research project.

\section{Competing interests}

The authors declare no competing financial interests.

\section{Additional information}

Supplementary information is available for this paper at https://doi.org/10.1038/ s41560-017-0054-3.

Reprints and permissions information is available at www.nature.com/reprints. Correspondence and requests for materials should be addressed to C.R.-C., G.M. or M.K.N.

Publisher's note: Springer Nature remains neutral with regard to jurisdictional claims in published maps and institutional affiliations. 